EUROPEAN ORGANIZATION FOR NUCLEAR RESEARCH

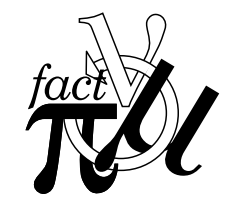

CERN NuFact Note 098

22 October 2001

\title{
Longitudinal capture of muons using bunch compression
}

\author{
B. Autin ${ }^{a}$ K. Bongardt ${ }^{\mathrm{a}, \mathrm{b}}$ J. Pasternak ${ }^{\mathrm{a}, \mathrm{c}}$ A. Verdier ${ }^{\mathrm{a}}$

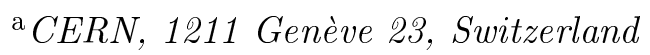 \\ ${ }^{\mathrm{b}}$ Forschungszentrum Juelich, Germany \\ ${ }^{\mathrm{c}}$ IFT, Wroclaw University, Poland
}

\begin{abstract}
When a particle beam drifts in a beam line, the bunch length increases due to the spread in particle velocity. Introducing a magnetic field so that the particles of high momentum follow a trajectory longer than the one of low momentum particles can limit the bunch extension and even compress it. This property is applied to the design of the longitudinal collection of a large emittance muon beam at $44 \mathrm{MHz}$ accelerating bucket.
\end{abstract}




\title{
Longitudinal capture of muons using bunch compression
}

\author{
B. Autin ${ }^{a}$ K. Bongardt ${ }^{a, b}$ J. Pasternak ${ }^{a, c}$ A. Verdier ${ }^{a}$

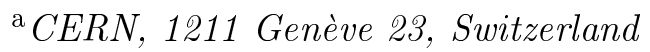 \\ ${ }^{\mathrm{b}}$ Forschungszentrum Juelich, Germany \\ ${ }^{\mathrm{c}}$ IFT, Wroclaw University, Poland
}

\begin{abstract}
When a particle beam drifts in a beam line, the bunch length increases due to the spread in particle velocity. Introducing a magnetic field so that the particles of high momentum follow a trajectory longer than the one of low momentum particles can limit the bunch extension and even compress it. This property is applied to the design of the longitudinal collection of a large emittance muon beam at $44 \mathrm{MHz}$ accelerating bucket.
\end{abstract}

\section{Introduction}

In the neutrino factory under investigation at CERN [1] trains of protons bunched at $44 \mathrm{MHz}[2]$ hit one or several heavy metal targets at a repetition frequency of $50 \mathrm{~Hz}$. The pions produced in the target are focused by magnetic horns either for a single or multiple beams [7] and injected into a solenoidal decay channel. At the end of the decay channel, pions have almost disappeared and are replaced by muons in a large momentum range. A big momentum spread is to be reduced to avoid excessive chromatic aberrations in the downstream systems. In the absence of effective cooling techniques in the longitudinal phase space, the bunches are rotated in the phase space to diminish the energy spread at the cost of a lengthening of the bunch imposed by Liouville theorem. The variant proposed in this paper consists of rotating the bunches in two stages separated by a magnetic compression [3-5]. The aim is to improve the longitudinal collection efficiency. The scheme is under design and consists of a solenoidal decay channel, a monochromator (Section 2), a magnetic compressor (Section 3) and a phase rotation (Section 4) which matches the beam to a cooling or accelerating RF system. 


\section{Decay channel}

The decay channel is essentially a $30 \mathrm{~m}$ long low $\beta$ insertion to prevent an excessive blow-up of the muon emittance due to the pion decay. The maximum transverse momentum of the muon in the center of mass frame is 29.79 $\mathrm{MeV} / \mathrm{c}$. After the Lorentz transformation into the laboratory system it should be small with respect to the pion transverse momentum. The solution applied in our study assumes an average $\beta$ value of $1 \mathrm{~m}$, see Fig(1) (left). The decay channel is located between the pion collector and the monochromator. The input $\beta$ value at the boundary is taken equal to $4 \mathrm{~m}$, which applies for single or multiple beams [7]. The same value of $\beta$ turned out to be well adopted to the monochromator. The two transition regions consist of a string of two solenoids of $0.7 \mathrm{~m}$ length, $30 \mathrm{~cm}$ aperture radius and $1.4 \mathrm{~T}$ magnetic field. In the low $\beta$ region, the corresponding parameters of the solenoids are 0.9 $\mathrm{m}, 15 \mathrm{~cm}$ and $2.4 \mathrm{~T}$. The pion beam at the beginning of the decay channel has the geometrical transverse emittance of $1.7 \mathrm{~cm} \mathrm{rad}, 4 \mathrm{~ns}$ long and has a total energy range $0.3-0.6 \mathrm{GeV}$ ( values taken from [8]) corresponding to the longitudinal portrait of $1.2 \mathrm{eVs}$ area.

\section{Monochromator}

After the decay channel the muon beam has larger transverse and longitudinal emittances compared with the initial pion beam. The system is designed to accept the geometrical transverse emittance of $2 \mathrm{~cm}$ rad and the area of the longitudinal portrait of $0.6 \mathrm{eVs}$. The central kinetic energy is fixed at 244 $\mathrm{MeV}$ with an energy range of $180 \mathrm{MeV}$ to contain maximum number of muons, which was calculated in [6], see Fig(1) (right). The normalized transverse emittance for the central energy is $6.3 \mathrm{~cm}$ rad. The monochromator has a regular structure of 9 periods. Each period contains two $1 \mathrm{~m}$ long $44 \mathrm{MHz}$ cavities separated by a solenoid of $0.6 \mathrm{~m}$ length and $1.5 \mathrm{~T}$ field. The $\beta$ value of $4 \mathrm{~m}$ about is determined by the $30 \mathrm{~cm}$ aperture radius of the cavities and a good packing factor for the RF systems of $2 / 3$ (we have two $1 \mathrm{~m}$ long RF cavities in each $3 \mathrm{~m}$ long period). The variations of the $\beta$ function along the decay channel and the monochromator are plotted in Fig(1) (left). The integrated electric field is $2 \mathrm{MV}$ per cavity. The longitudinal phase space portrait corresponding to the assumed values is given in Fig(2) (upper left). Please note the tilted longitudinal distribution due to about $40 \mathrm{~m}$ distance from the pion production target. The energy spread is reduced from $155-335 \mathrm{MeV}$ to $190-$ $295 \mathrm{MeV}$, shown in Fig(2) (upper right). The time width of $12 \mathrm{~ns}$ is outside the acceptance of an accelerating $44 \mathrm{MHz}$ bucket. The particle tracking in the monochromator and a matching triplet needed for the magnetic compression system in the horizontal and the vertical plane are shown in the lower part of 
$\operatorname{Fig}(2)$.
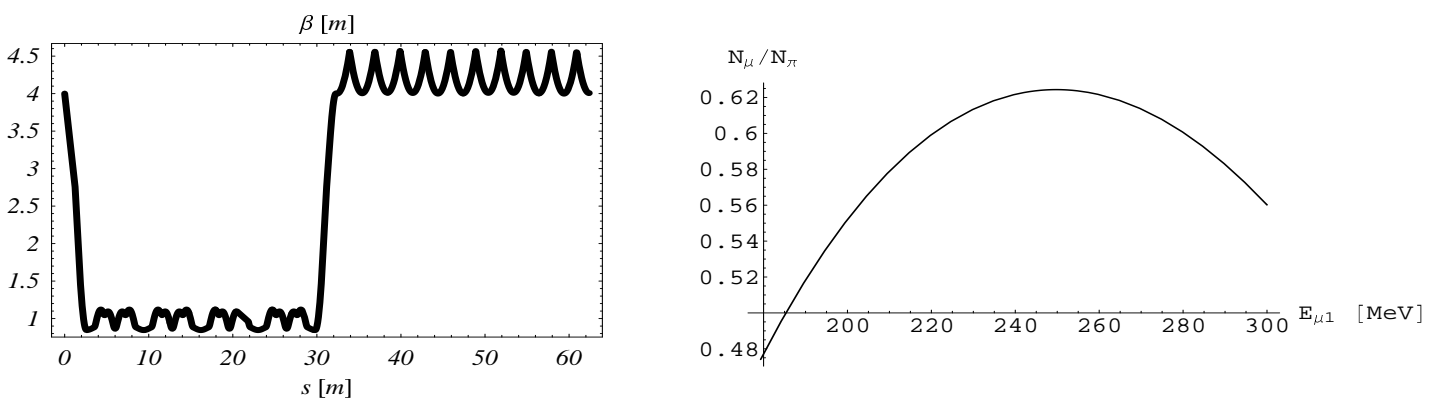

Fig. 1. Variations of $\beta$ in the decay channel and in the first monochromator including transition regions (left). Variation of the relative number of collected muons within a $160 \mathrm{MeV}$ range as a function of the lowest muon energy (right).
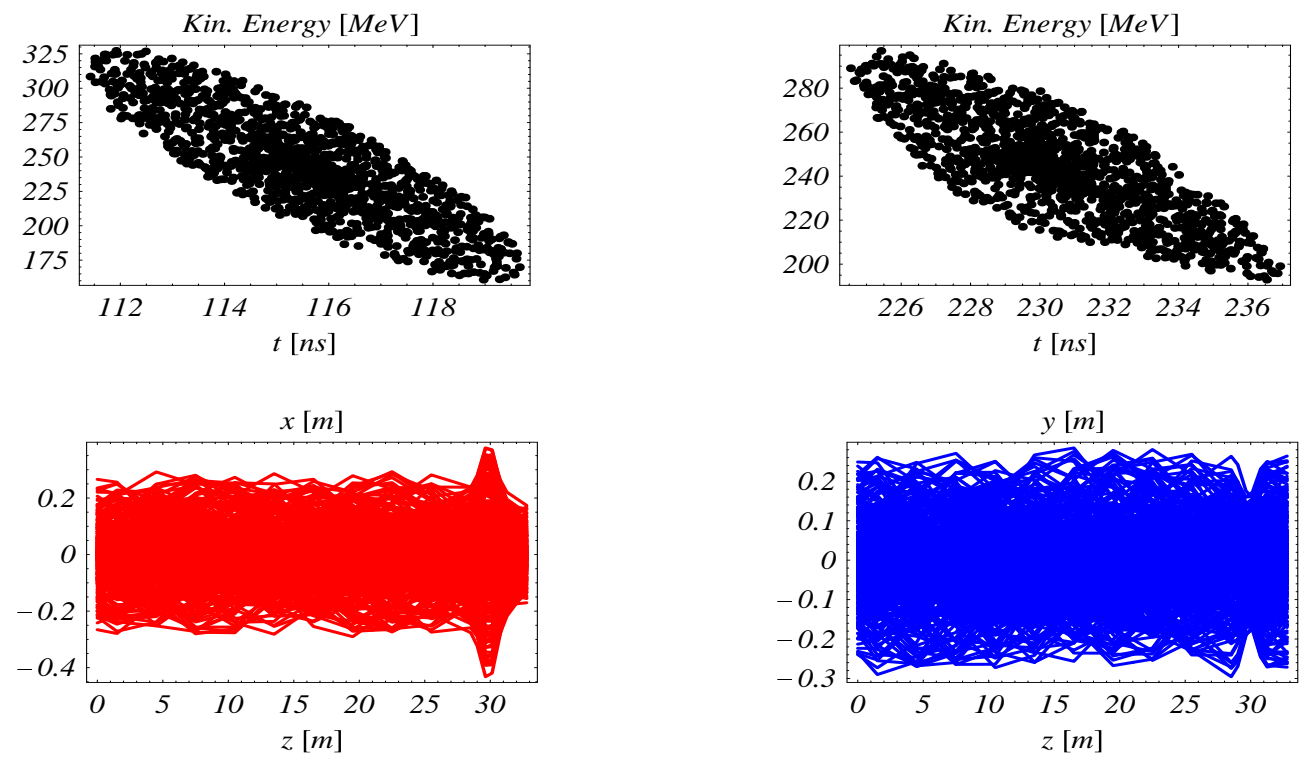

Fig. 2. Longitudal portrait assumed befor (upper left) and after (upper right) of the monochromator (phase rotation 1). The particle tracking in the monochromator in the horizontal (lower left) and in the vertical (lower right) plane.

\section{Magnetic compression}

The design principle of a magnetic compression is to modify the length for off-momentum particles so that the fast particle travel on a long orbit and the slow particle on a short orbit. The following relation gives the dependence of the relative transit time on the lattice properties for given momentum spread:

$$
\Delta t / t=\left(\alpha_{p}-\gamma^{-2}\right) \Delta p / p
$$


where $\alpha_{p}$ is the momentum compaction factor and $\gamma$ the relativistic factor for the bunch center. In order to better fit the beam longitudinaly to a $44 \mathrm{MHz}$ accelerating RF bucket, the goal is to achieve bunch compression by a factor of 2 in time. On the downstream side of the monochromator the beam enters the $16 \mathrm{~m}$ long magnetic compressor lattice shown on the left hand side of the $\operatorname{Fig}(3)$.
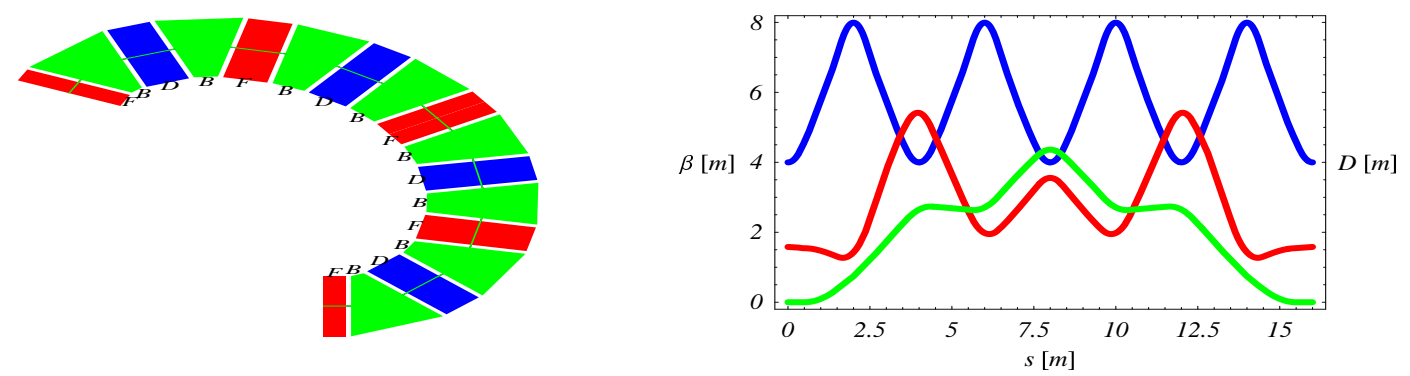

Fig. 3. The compressor lattice (left), variation of beta functoins and dispersion in the compressor (right). In particular the dispersion vanishes at the beginning and at the end of the compressor.

Due to the introduction of dipolar fields in the beam line $\eta$ is equal to 0.35 for a relativistic gamma of 3.3 (244 MeV kinetic energy). The compressor lattice is isochronus at $\gamma=1.5$. The optical functions are shown in Fig(3). The magnetic gradient $(0.56 \mathrm{~T} / \mathrm{m})$ is constant in the compressor quadrupoles and corresponds to a maximum field of $0.36 \mathrm{~T}$. The dipole field is limited to 0.85 T. To match solenoidal lattices before and after the compressor, quadrupole triplets are added to the compressor. In Fig(4) we show an example, which shows evolution of the bunch in the compressor. An idealized elliptical distribution in the longitudinal plane being perfectly matched in the transverse planes is shown on the left of Fig(4) enters the compressor lattice. The final distribution at the exit of the compressor is shown on the right together with the elliptical boundary of the initial distribution. From this figure the reduction of the bunch length with constant bunch area and energy spread is visible. One can also see an asymmetry in the compression between low and high energy part of the bunch indicating that there is need for further improvements.

The tracking of the realistic particle distribution coming from the monochromator and the matching section is shown in Fig(5). One can see the large apperture in the horizontal plane with up to $65 \mathrm{~cm}$ in radius. 

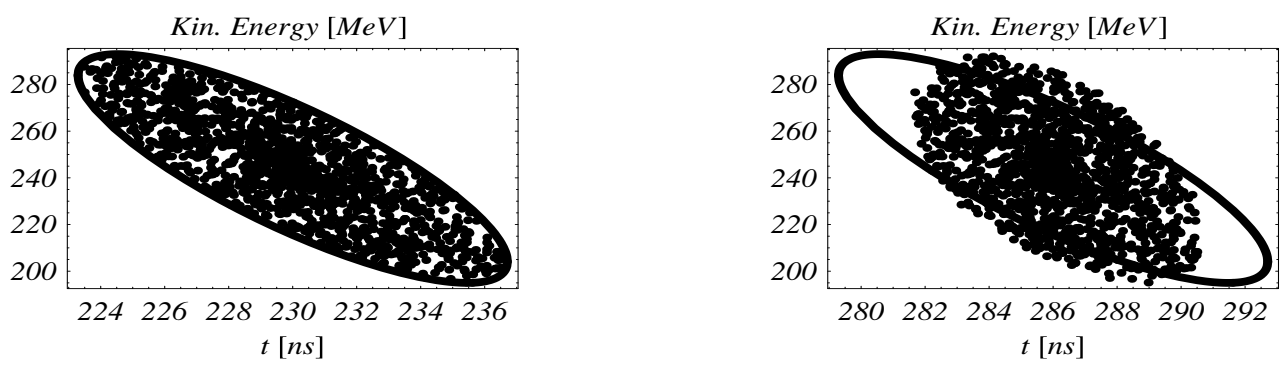

Fig. 4. The results of tracking of an idealized distribution (left) and the obtained output one (right) in the compressor. The eliptical boundaries shown on both sides are identical.
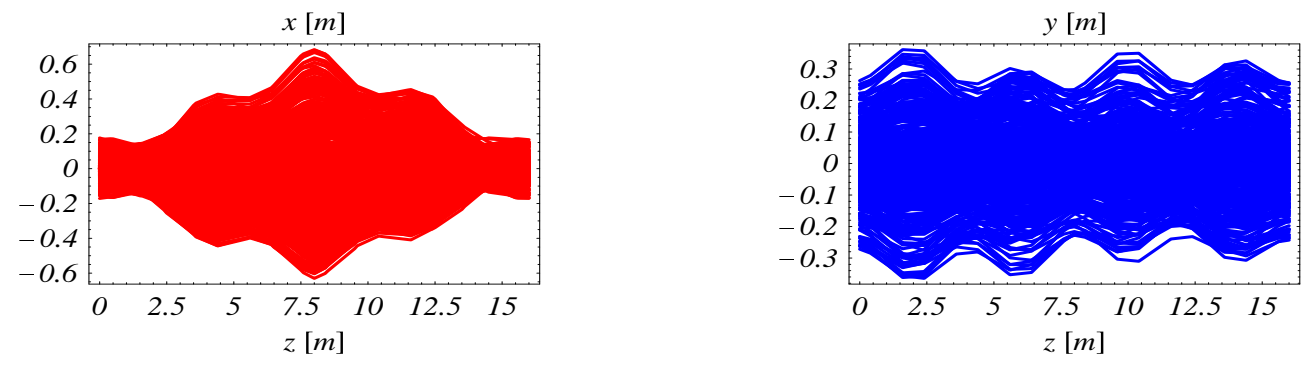

Fig. 5. The particle tracking in the compressor in the horizontal (left) and vertical (right) plane.

\section{Phase rotation}

The section of the phase rotation matches the bunch into the bucket of the next accelerator. It is made of 7 periods identical to those of the monochromator. Transverse tracking in the second monochromator is shown in the upper part of Fig(6). Obvious is some mismatch in the vertical plane, see Fig(6) (upper right) . The Fig(6) (lower left) shows the results of beam matching with the help of the monochromator, the compressor and the phase rotation section (the second monochromator) enclosed by the separatrix corresponding to a $44 \mathrm{MHz}$ bucket, a $\pi / 4$ synchronous phase, an accelerating electric field of $2 \mathrm{MV} / \mathrm{m}$ and an area of $0.52 \mathrm{eVs}$. The lower right side of Fig(6) shows results of tracking in a lattice without magnetic compression, consists of the monochromator followed by the phase rotation section. The comparison of left and right hand sides of the lower part of Fig(6) shows an advantage offered by a magnetic compression, which enables to match the beam to the bucket. 

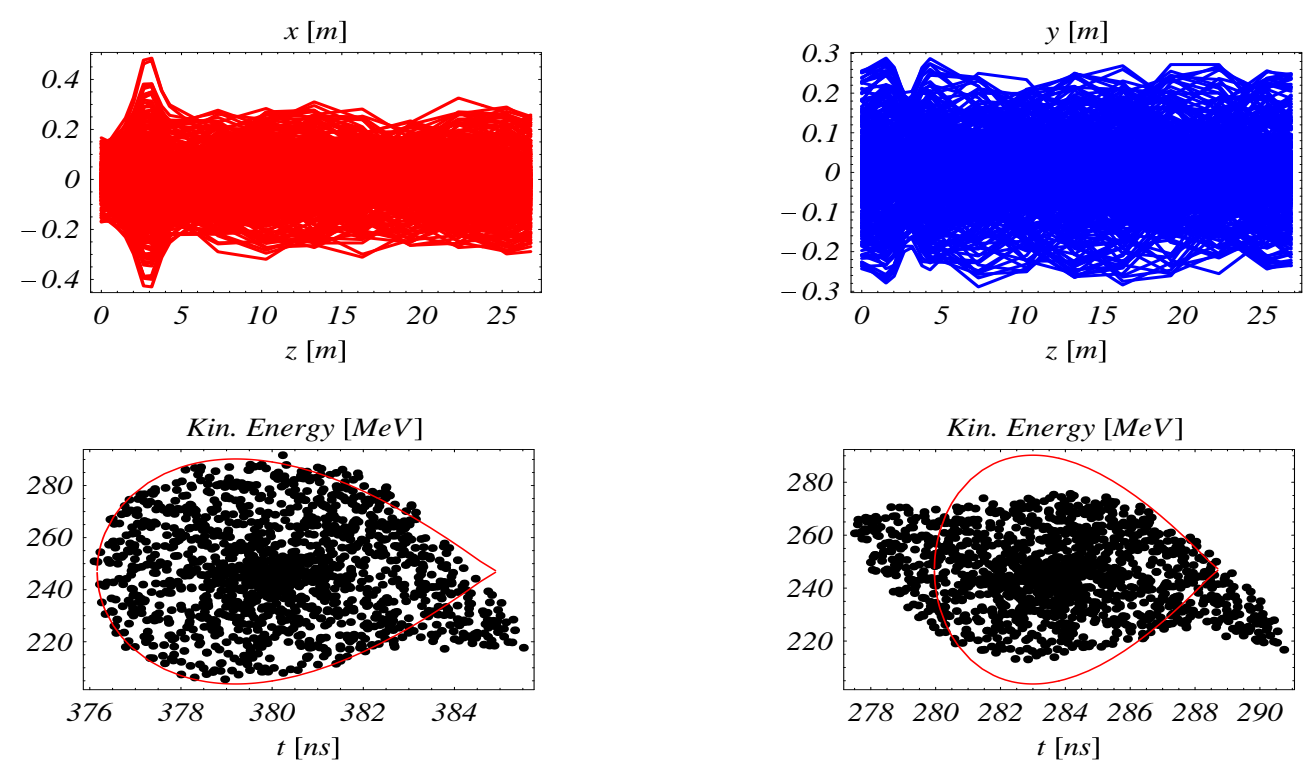

Fig. 6. The particle tracking in the matching triplet and the phase rotation section (the second monochromator) in the horizontal (upper left) and vertical (upper right) plane. Longitudal portrait showing the final matching to $44 \mathrm{MHz}$ accelerating RF bucket after phase rotation 2 (lower left) and longitudinal portrait without compressor (lower right).

\section{Conclusion}

The combination of the RF phase rotation and the magnetic bunch compression can be used to improve the longitudinal collection of muons, which is an important task to achieve a high neutrino flux. The compressor allows to match the muon bunch to the $44 \mathrm{MHz}, 2 \mathrm{MV}$ accelerating RF bucket, which can be used for acceleration or cooling. Even for large 6D emittance, the compressor can be designed by the technique of alternating gradient lattices.

\section{References}

[1] B. Autin at al., The CERN Neutrino Factory Working Group. Status Report and Work Plan, CERN-NUFACT Note Nr 28.

[2] R. Garoby, F. Gerigk, Cavity design for the CERN muon cooling channel, CERN-NUFACT Note Nr 87.

[3] T. O. Raubenheimer, P. Emma, S. Kheifets, Chicane and Wiggler Based Bunch Compressors for Future Linear Colliders,SLAC-PUB 6119 (1993)

[4] T. E. D'Amico, G. Guignard, T. Raubenheimer, CLIC Note Nr. 2 (1998) 
[5] B. Autin, M. Giovannozi,M. Martini, Ph. Royer, Varying the Path Length of Charged Particles Using Wiggler Modules, CERN-NUFACT Note Nr 69.

[6] B. Autin, Muon emittance and pion decay, These Proceedings.

[7] B. Autin, A. Verdier, Multiple pion beam collection for a neutrino factory, these Proceedings.

[8] B. Autin, S. Gilardoni, P. Sievers, Conducting target for pion production, these Proceedings. 